\title{
Epidemiology of tuberculosis in the EU/EEA in 2010 - monitoring the progress towards tuberculosis elimination
}

A Sandgren (Andreas.Sandgren@ecdc.europa.eu) ${ }^{1}$, V Hollo $^{1}$, E Huitric ${ }^{1}$, C Ködmön $^{1}$

1. European Centre for Disease Prevention and Control (ECDC), Stockholm, Sweden

Citation style for this article:

Sandgren A, Hollo V, Huitric E, Ködmön C. Epidemiology of tuberculosis in the EU/EEA in 2010 - monitoring the progress towards tuberculosis elimination. Euro Surveill. 2012;17(12):pii=20124. Available online: http://www.eurosurveillance.org/ViewArticle.aspx?Articleld=20124

Article published on 22 March 2012

The 2012 combined tuberculosis (TB) surveillance and monitoring report for the European Union and European Economic Area identifies a mean annual decline in TB notification rate by $4.4 \%$ from 2006 to 2010. Culture confirmation for new pulmonary cases and drug susceptibility testing have increased to $65.6 \%$ and $70.8 \%$, but remain under their targets of $80 \%$ and $100 \%$, respectively. Reporting of treatment outcome and coinfection with human immunodeficiency virus also remain suboptimal. Strengthened control practices are needed to allow progress towards TB elimination.

\section{Monitoring progress towards tuberculosis elimination through surveillance}

Surveillance is an essential element in monitoring the effectiveness of interventions aimed at controlling and eliminating tuberculosis (TB). It is one of the eight strategic areas of the Framework Action Plan to Fight TB in the European Union (EU) which was launched in 2008 by the European Centre for Disease Prevention and Control (ECDC) [1]. Since 1 January 2008, the ECDC and the World Health Organization (WHO) Regional Office for Europe have been jointly coordinating the TB surveillance activities in Europe and publishing a joint annual TB surveillance report [2]. In 2010, ECDC launched the follow-up to the Framework Action Plan: an epidemiological and strategic monitoring framework that allows progress towards TB elimination in the EU to be assessed [3]. The follow-up monitoring framework entails four epidemiological indicators calculated from individual case data and eight core operational indicators (Table 1). The monitoring is focused on giving an overview of the progress made towards elimination, by presenting the status and trends of the specific indicators for the EU and European Union and European Economic Area (EEA) as a whole and for the individual countries. The epidemiological trends are expressed as mean annual percentage changes, which are calculated over a period of either five or 10 years, to avoid the effect of random variation over time [3]. Starting this year, 2012, these epidemiological and core operational indicators are being monitored and are presented in the TB surveillance and monitoring report for Europe [4]. The 2012 report covers data for the TB cases notified in the 53 countries of the WHO European Region in 2010. This rapid communication presents an overview of the monitoring aspects of the report for the 27 EU Member States, Iceland and Norway (Table 2).

\section{Tuberculosis situation in the EU/EEA in 2010}

In 2010, 73,996 TB cases were reported by the $27 \mathrm{EU}$ Member States, Iceland and Norway. The overall notification rate in 2010 was 14.6 per 100,000 population, with a mean annual decline in the case notification rate of $4.4 \%$ during the period 2006 to 2010 . The target of reaching a mean decline over five years was met by the EU/EEA overall and by 22 of the 29 Member States. For the first time, all EU/EEA Member States had notification rates below 100 per 100,000 population and one additional country, Poland, joined the 22 countries already in the elimination phase defined as below 20 cases per 100,000 [5].

\section{Resistant tuberculosis}

Resistance to at least the first-line anti-TB drugs isoniazid and rifampicin was reported for, respectively, $1,374(7.8 \%)$ and 529 (3.0\%) of 17,559 new pulmonary TB cases tested for drug susceptibility in the EU/EEA. Three countries did not report, four countries did not have any isoniazid-resistant cases and six countries did not have any rifampicin-resistant cases. Drug susceptibility test results were known for $70.8 \%$ of the 24,785 new pulmonary culture-positive cases. All countries reported and four countries did not have any cases. The proportion of multi-drug resistant TB (MDR-TB, defined as resistance to at least isoniazid and rifampicin) among all culture-positive TB cases was $4.6 \%$ in the 29 reporting countries. This proportion corresponded to a mean five-year decline of $3.4 \%$ for the period 2006 to 2010 , with seven of 22 countries reaching the set target of a declining trend. In 2010, 108 (13.2\%) of the 819 MDR-TB cases tested for secondline drug resistance were reported to be extensively 
TABLE 1

Indicators for monitoring progress of the Framework Action Plan to Fight TB in the European Union

Epidemiological indicators

1. Trends in case notification rate,

2. Trends in multidrug-resistant case notification rate,

3. Trends in ratio of notification rates in children versus adults,

4. Trends in mean age of TB cases.

Operational indicators

1. Availability of a national TB control plan,

2. Availability of guidelines for implementing the national TB control plan,

3. Percentage of European TB reference laboratory network members achieving adequate performance in the external quality assurance scheme,

4. Availability of a strategy for introducing and implementing new tools for TB control,

5. Percentage of new pulmonary TB cases confirmed by culture and percentage of cases tested for susceptibility to first-line drugs,

6. Percentage of EU Member States reporting treatment success rate,

7. Treatment success rate,

8. Percentage of TB patients for whom HIV status is known.

EU: European Union; HIV: human immunodeficiency virus; TB: tuberculosis.

Source: This Table has been adapted from [3].

\section{TABLE 2}

Monitoring of the follow-up to the Framework Action Plan to fight TB in the EU, 2010

\begin{tabular}{|c|c|c|c|c|}
\hline Indicator & Target & $\begin{array}{l}\text { EU/EEA } \\
\text { status }\end{array}$ & $\begin{array}{c}\text { Number of Member States } \\
\text { reaching the target }\end{array}$ & $\begin{array}{c}\text { Number of Member } \\
\text { States reporting }\end{array}$ \\
\hline \multicolumn{5}{|l|}{ Epidemiological indicators } \\
\hline Trend in TB case notification rate & Mean five-year decline & $-4.4 \%^{\mathrm{a}}$ & 22 & 29 \\
\hline Trend in MDR-TB case notification rate & Mean five-year decline & $-3.4 \%^{a}$ & 7 & 22 \\
\hline $\begin{array}{l}\text { Trend in ratio of notification rate in } \\
\text { children versus adults }\end{array}$ & Mean 10-year decline & $-0.3 \%{ }^{a}$ & 7 & 25 \\
\hline Trend in mean age of TB cases $^{b}$ & Increasing trend over 10 years & $0.0 \%^{a}$ & 10 & 24 \\
\hline \multicolumn{5}{|l|}{ Core indicators } \\
\hline Availability of a National TB Planc & TB Plan available for all countries & $50.0 \%$ & 14 & 28 \\
\hline Availability of TB Guidelines & TB Guidelines available & Not collected & Not done & Not done \\
\hline Laboratory EQA performance $^{d}$ & $\begin{array}{c}100 \% \text { reference TB laboratories } \\
\text { achieving } 80 \% \text { performance } \\
\text { (smear, culture, DST) }\end{array}$ & $79.0 \%$ & $N A^{e}$ & 23 \\
\hline Availability of a new tool strategy & Strategy available & Not collected & Not done & Not done \\
\hline Culture confirmation & $\begin{array}{l}80 \% \text { culture confirmation in new } \\
\text { pulmonary cases. }\end{array}$ & $65.6 \%{ }^{a}$ & 12 & 29 \\
\hline DST results of new pulmonary cases & $\begin{array}{c}100 \% \text { DST results to first-line drugs } \\
\text { among new pulmonary culture- } \\
\text { positive cases }\end{array}$ & $70.8 \%^{a}$ & 8 & 26 \\
\hline $\begin{array}{l}\text { Proportion of Member States } \\
\text { reporting treatment outcome }\end{array}$ & $100 \%$ & $82.8 \%$ & $N A^{e}$ & 24 \\
\hline Treatment success rate & $\begin{array}{c}85 \% \text { in new pulmonary culture- } \\
\text { positive cases }\end{array}$ & $78.8 \%^{a}$ & 4 & 24 \\
\hline Treatment success for MDR-TB & $70 \%$ in new pulmonary MDR-TB & $49.3 \%^{a}$ & 4 & 16 \\
\hline Proportion with known HIV status & $\begin{array}{l}\text { HIV status known for } 100 \% \text { of TB } \\
\text { cases }\end{array}$ & $23.9 \%^{a}$ & 1 & 15 \\
\hline
\end{tabular}

DST: drug susceptibility testing; EEA: European Union and European Economic Area; EQA: external quality assessment; HIV: human immunodeficiency virus; MDR-TB: multidrug-resistant tuberculosis; NA: not applicable; TB: tuberculosis.

a Data originating from individual case data.

b Crude mean age used for calculations.

c Results obtained from 2009 survey.

d Information is available only for DST to isoniazid and rifampicin, data currently obtained from International EQA scheme. In the future, these data will be obtained from the European Reference Laboratory Network for TB (ERLN-TB).

e EU level indicator, no trend involved.

Source: This Table has been adapted from [4]. 
drug-resistant (XDR-TB, defined as resistance to isoniazid, rifampicin, any fluoroquinolone and at least one of the injectable second-line drugs capreomycin, kanamycin or amikacin).

\section{Childhood tuberculosis}

In 2010, 3,035 TB cases were reported in children (under the age of 15 years), accounting for $4.1 \%$ of all notified cases. The trend in childhood TB gives an indirect measure of the level of transmission in the community [6]. The trend in mean age of TB cases is another estimate of how effective the TB control is in interrupting transmission in the community [3]. Overall, the ratio of notification rates in children versus adults declined by $0.3 \%$ over the period 2001 to 2010 , and the mean age did not change at all in the same period with 45.0 years for 2001 and 45.1 years for 2010. There was, however, variation across countries, in that seven of 25 reporting countries met the target of a declining trend in the ratio of cases in children versus adults and 10 of 24 reporting countries met the target of an increasing trend in mean age.

Operational strategies, policies and practices A national TB control plan was available in 14 of the 28 countries that had responded to a survey ECDC conducted for this purpose in 2009 [4]. No information has been collected yet about the availability of guidelines for implementing the national TB control plan or the availability of strategies for introducing and implementing new tools for TB control. The European Reference Laboratory Network for TB [7] is currently conducting external quality assurance (EQA) in laboratory proficiency for smear microscopy, culture and drug susceptibility testing (DST) for first- and second- line anti-TB drugs, thus the results are not yet available and not presented in this year's report. Twenty-three national reference laboratories reported on performance in DST for first-line anti-TB drugs following the international EQA schemes of the WHO Supra-National Reference Laboratory Network. All 23 laboratories reported full agreement of results, demonstrating high-quality DST.

\section{Bacteriological confirmation of cases}

Overall, $65.6 \%$ of new pulmonary TB cases were culture-confirmed. At country level, only 12 of 29 countries achieved the $80 \%$ culture-confirmation target among new pulmonary TB cases. Likewise, only eight of 26 countries achieved the target of testing $100 \%$ of new pulmonary culture-positive cases for susceptibility to first-line drugs. For the EU/EEA as a whole, 70.8\% of culture-positive new pulmonary TB cases had DST results for first-line drugs available.

\section{Treatment outcome}

In the 2009 treatment cohort, 24 of the 29 countries reported on treatment outcome, falling short of the target of having all EU/EEA Member States reporting treatment outcome data. Seven countries reported treatment outcome for all cases. The treatment success rate was $78.8 \%$ for new pulmonary culture-positive cases, with only four of 24 countries reaching the target of more than $85 \%$ treatment success. Treatment success of new pulmonary culture-positive MDR-TB cases in the 2008 cohort was $49.3 \%$, with only four of 16 reporting countries reaching the target (taking into consideration only countries reporting at least one MDR-TB culture-positive pulmonary case).

\section{Human immunodeficiency virus co-}

\section{infection in tuberculosis cases}

Most EU/EEA Member States have not incorporated human immunodeficiency virus (HIV) testing for TB patients in the national plans or do not report HIV status. The target is that HIV status should be known for $100 \%$ of the TB cases. Overall in the EU/EEA, only $23.9 \%$ of the TB patients have a known HIV status. Only one of 15 reporting countries reached the $100 \%$ target.

\section{Conclusions}

These data demonstrate that most EU/EEA Member States have continued to experience a steady decrease in the overall TB notification rate during 2010. Several challenges remain, however, that need to be addressed. The proportions of bacteriologically confirmed TB cases and cases for which drug-susceptibility testing has been performed are increasing, but remain suboptimal in the EU/EEA, thus laboratory practices need to be further strengthened. Efforts are also needed to improve the reporting of treatment outcomes and to ensure successful treatment of new culture-confirmed TB and MDR-TB cases; for example only four of 24 countries reached the $85 \%$ target of treatment success among new pulmonary culture-positive cases. Further, reporting of HIV co-infection is lacking in many countries, indicating that national TB programmes are lacking targeted, incorporated TB/HIV plans. Monitoring overall EU/EEA trends can mask patterns for some indicators, in particular for mean age of TB cases and the ratio of notification rates in children versus adults, therefore some indicators may be more relevant to monitor at Member State level. We therefore encourage similar analyses to be performed at country level when possible. For the monitoring of progress towards TB elimination to be a valid tool, a surveillance system that captures close to $100 \%$ of all TB cases is a prerequisite. Thus, the monitoring framework might also be a tool for further improving the quality and coverage of surveillance systems.

\section{Acknowledgments}

The authors would like to thank all the nominated TB experts of the EU/EEA Member States for providing the surveillance data to TESSy. These are:

Ibrahim Abubakar, Peter Henrik Andersen, Delphine Antoine, Wouter Arrazola de Oñate, Thorsteinn Blondal, Paul Caruana, Nicoleta Cioran, Edita Davidaviciene, Francis Drobniewski, Connie Erkens, Jos Even, Lanfranco Fattorini, Maryse Fauville Dufaux, António Fonseca Antunes, Walter Haas, Chrystalla Hadjianastassiou, Sven Hoffner, Vincent Jarlier, Maria Soledad Jimenez Pajares, Jerker Jonsson, Jean - Paul Klein, Maria Korzeniewska - Kosela, Tiina Kummik, Daniel LévyBruhl, Turid Mannsåker, Merja Marjamäki, Tanya Melillo 
Fenech, Vladimir Milanov, Olga Moldovan, Joan O’Donnell, Vibeke Østergaard Thomsen, Analita Pace-Asciak, Despo Pieridou Bagatzouni, Erik Michael Rasmussen, Vija Riekstina, Elena Rodríguez Valín, Thomas Rogers, Karin Rønning, Sabine Rüsch-Gerdes, Petri Ruutu, Girts Škenders, Pavel Slezák, Ivan Solovic, Anaida Sosnovskaja, János Strausz, Petra Svetina - Sorli, Nora Szabó, Wim Van Der Hoek, Dick Van Soolingen, Piret Viiklepp, Jiri Wallenfels, Maryse Wanlin, John Watson, Ilona Zemanova, Manca Zolnir-Dovc, Zofia Zwolska.

\section{References}

1. European Centre for Disease Prevention and Control (ECDC). Framework action plan to fight tuberculosis in the European Union. Stockholm: ECDC; Feb 2008. Available from: http:// www.ecdc.europa.eu/en/publications/Publications/0803_ SPR_TB_Action_plan.pdf

2. Hollo V, Zucs P, Ködmön C, Sandgren A, Manissero D. Marking 15 years of efforts towards a comprehensive European TB surveillance system: the epidemiological situation of TB in the EU/EEA in 2009. Euro Surveill. 2011;16(12): $\mathrm{ii}=19822$. Available from: http://www.eurosurveillance.org/ViewArticle. aspx?Articleld $=19822$

3. European Centre for Disease Prevention and Control (ECDC). Progressing towards TB elimination. Stockholm: ECDC; Nov 2010. Available from: http://www.ecdc.europa.eu/en/ publications/Publications/101111_SPR_Progressing_towards TB_elimination.pdf

4. European Centre for Disease Prevention and Control (ECDC)/ World Health Organization Regional Office for Europe. Tuberculosis surveillance and monitoring in Europe 2012. Stockholm: ECDC; Mar 2012. Available from: http://ecdc. europa.eu/en/publications/Publications/1203-Annual-TBReport.pdf

5. Broekmans JF, Migliori GB, Rieder HL, Lees J, Ruutu P, Loddenkemper R, et al. European framework for tuberculosis control and elimination in countries with a low incidence. Recommendations of the World Health Organization (WHO), International Union Against Tuberculosis and Lung Disease (IUATLD) and Royal Netherlands Tuberculosis Association (KNCV) Working Group. Eur Respir J. 2002;19(4):765-75.

6. Sandgren A, Hollo V, Quinten C, Manissero D. Childhood tuberculosis in the European Union/European Economic Area, 2000 to 2009. Euro Surveill. 2011;16(12):pii=19825. Available from: http://www.eurosurveillance.org/ViewArticle. aspx?Articleld $=19825$

7. Drobniewski FA, Nikolayevskyy V, Hoffner S, Pogoryelova O, Manissero D, Ozin AJ. The added value of a European Union tuberculosis reference laboratory network - analysis of the national reference laboratory activities. Euro Surveill. 2008;13(12):pii=8076. Available from: http://www. eurosurveillance.org/ViewArticle.aspx?Articleld $=8076$ 